\title{
BMJ Open Surgical treatment for colorectal cancer: analysis of the influence of an enhanced recovery programme on long-term oncological outcomes - a study protocol for a prospective, multicentre, observational cohort study
}

To cite: Ramirez-Rodriguez JM, Martinez-Ubieto J, MuñozRodes J-L, et al. Surgical treatment for colorectal cancer: analysis of the influence of an enhanced recovery programme on long-term oncological outcomes-a study protocol for a prospective, multicentre, observational cohort study. BMJ Open 2020;10:e040316. doi:10.1136/ bmjopen-2020-040316

- Prepublication history for this paper is available online. To view these files, please visit the journal online (http://dx.doi. org/10.1136/bmjopen-2020040316).

Received 11 May 2020

Revised 15 August 2020

Accepted 30 September 2020

Check for updates

(C) Author(s) (or their employer(s)) 2020. Re-use permitted under CC BY-NC. No commercial re-use. See rights and permissions. Published by BMJ.

For numbered affiliations see end of article.

Correspondence to

Professor Jose-M Ramirez-

Rodriguez; jramirez@unizar.es

Jose-M Ramirez-Rodriguez (1) , , ${ }^{1,2}$ Javier Martinez-Ubieto, ${ }^{3}$ Jose-L Muñoz-Rodes, ${ }^{4}$ Jose-R Rodriguez-Fraile, ${ }^{5}$ Jose-A Garcia-Erce, ${ }^{6}$ Javier Blanco-Gonzalez, ${ }^{7}$ Emilio Del Valle-Hernandez, ${ }^{8}$ Alfredo Abad-Gurumeta, ${ }^{9}$ Eugenia Centeno-Robles, ${ }^{10}$ Carolina Martinez-Perez, ${ }^{11}$ Miguel Leon-Arellano, ${ }^{12}$ Estibaliz Echazarreta-Gallego, ${ }^{13}$ Manuela Elia-Guedea, ${ }^{1}$ Ana Pascual-Bellosta, ${ }^{3}$ Elena Miranda-Tauler, ${ }^{4}$ Alba Manuel-Vazquez, ${ }^{5}$ Enrique Balen-Rivera, ${ }^{6}$ David Alvarez-Martinez, ${ }^{7}$ Jose Perez-Peña, ${ }^{8}$ Ane Abad-Motos, ${ }^{9}$ Elisabeth Redondo-Villahoz, ${ }^{10}$ Elena Biosta-Perez, ${ }^{11}$ Hector Guadalajara-Labajo, ${ }^{12}$ Javier Ripollés-Melchor, ${ }^{9}$ Cristina Latre-Saso, ${ }^{13}$ Elena Cordoba-Diaz de Laspra, ${ }^{1}$ Luis Sanchez-Guillen, ${ }^{4}$ Mercedes Cabellos-Olivares, ${ }^{5}$ Javier Longas-Valien, ${ }^{1}$ Sonia Ortega-Lucea, ${ }^{3}$ Julia Ocon-Breton, ${ }^{1}$ Antonio Arroyo-Sebastian, ${ }^{4}$ Damian Garcia-Olmo ${ }^{12,14}$

\section{ABSTRACT}

Introduction The evidence currently available from enhanced recovery after surgery (ERAS) programmes concerns their benefits in the immediate postoperative period, but there is still very little evidence as to whether their correct implementation benefits patients in the long term. The working hypothesis here is that, due to the lower response to surgical aggression and lower rates of postoperative complications, ERAS protocols can reduce colorectal cancer-related mortality. The main objective of this study is to analyse the impact of an ERAS programme for colorectal cancer on 5-year survival. As secondary objectives, we propose to analyse the weight of each of the predefined items in the oncological results as well as the quality of life.

Methods and analysis A multicentre prospective cohort study was conducted in patients older than 18 years of age who are scheduled to undergo surgery for colorectal cancer. The study involved 12 hospitals with an implemented enhanced recovery protocol according to the guidelines published by the Spanish National Health Service. The intervention group includes patients with a minimum implementation level of $70 \%$, and the control group includes those who fail to reach this level. Compliance will be studied using 18 key performance indicators, and the results will be analysed using cancer survival indicators, including overall survival, cancerspecific survival and relapse-free survival. The time to recurrence, perioperative morbidity and mortality, hospital stay and quality of life will also be studied, the latter using

\section{Strengths and limitations of this study}

- This is the first multicentre prospective study intending to analyse whether the correct implementation of an intensified recovery programme (enhanced recovery after surgery) in patients undergoing colorectal surgery for cancer is related to better long-term oncological outcomes.

- The study will also try to analyse the influence (weight) of each perioperative protocol items on the oncological outcome.

- The research project will be monitored closely by a certified external auditor to ensure that study activities are carried out in accordance with the protocol, good clinical practice and applicable regulatory requirements. Data quality will also be audited.

- The study is designed as a prospective, nonrandomised study.

- The study could have difficulty in recruiting patients due to potential structural or multidisciplinary team problems.

the validated EuroQol Five questionnaire. The propensity index method will be used to create comparable treatment and control groups, and a multivariate regression will be used to study each variable. The Kaplan-Meier estimator will be used to estimate survival and the log-rank test to make comparisons. A p value of less than 0.05 (two-tailed) will be considered to be significant. 
Ethics and dissemination Ethical approval for this study was obtained from the Aragon Ethical Committee (C.P.-C.I. PI20/086) on 4 March 2020. The findings of this study will be submitted to peer-reviewed journals (BMJ Open, JAMA Surgery, Annals of Surgery, British Journal of Surgery). Abstracts will be submitted to relevant national and international meetings.

Trial registration number NCT04305314.

\section{INTRODUCTION}

Colorectal cancer is the third most frequent neoplasm in men worldwide (746000 cases, approximately $10.0 \%$ of the total) and the second most common in women (614000 cases, 9.2\%). Surgery remains a cornerstone of treatment for this type of malignant tumour. ${ }^{1}$ In this regard, surgical treatment with curative intent is indicated in about $80 \%$ of cases, with a 5 -year overall survival rate of approximately $65 \% .^{2}$ It is well known that any surgical procedure can lead to adverse events, with surgery-related complications depending on the degree of aggression (stress), the basal state of the patient and the disease itself. Postoperative complications after major surgery have been shown to increase both the length of hospital stay and cost while decreasing long-term survival as an independent factor. ${ }^{34}$ Colorectal surgery is considered a high risk and is associated with a high rate of morbidity and mortality in the immediate postoperative period. Despite 'curative intent' surgery, 5-year survival in colorectal cancer has remained stable at around $60 \%$ in recent decades. Metastatic disease is the most important cause of cancer-related death in patients after surgery. ${ }^{5}$ Although there has been much speculation about the occurrence of metastasis, surgical manipulation is known to lead to significant systemic release of tumour cells. ${ }^{67}$ Whether these cells lead to metastasis depends largely on the balance between the aggressiveness of the tumour cells and the resistance of the patient. As we have discussed previously, surgery 'per se' induces a stress response that can decrease host defences and promote tumour growth. Furthermore, innate immunity and, especially, natural killer (NK) cells are known to play an important role in the elimination of circulating tumour cells. ${ }^{8}$ Several studies have shown decreased postoperative NK cell activity and an inverse correlation of NK cell activity with tumour stage and metastatic growth. ${ }^{9}$

Until a few years ago, the perioperative treatment of patients undergoing elective abdominal surgery consisted of a series of habits acquired by practice rather than scientifically proven facts. In the early 2000s, ERAS (enhanced recovery after surgery) protocols based mainly on Kehlet's work began to be introduced in some centres. These programmes rest on three fundamental pillars: the application of a package of perioperative measures and strategies; interdisciplinarity, understood to be the joint and structured participation of the various health professionals involved; and active participation of the patient throughout the process. The various ERAS protocol recommendations include anaesthesia/analgesia, goaldirected fluid therapy, prevention of nausea and ileus, thromboembolic prophylaxis, minimally invasive techniques, temperature control, early nutrition and early mobilisation.

A number of randomised studies and meta-analyses carried out in the first decade of this century served to demonstrate that these protocols both shorten the hospital stay and decrease complications, outcomes that have been linked to the amelioration of perioperative care and the reduction in the response to surgical stress. ${ }^{1011}$

As mentioned previously, the response to surgical stress results in hormonal and metabolic changes that produce immune and endocrine responses proportionally to the extent of the surgical tissue injury. Local changes affect the inflammatory reaction throughout the body, leading to widespread effects on organ function and the development of complications. In this regard, numerous studies have pointed out that the main reason for the effectiveness of ERAS programmes is based on the ability of each element to reduce the stress response to injuries and maintain homeostasis. ${ }^{12}$ Hence, prevention of the stress response is the key mechanism underlying perioperative ERAS programmes. ${ }^{13}$ Moreover, since a lower surgical aggression has evident advantages in the immunometabolic response of the patient with cancer, it could be deduced that, in these cases, long-term survival is favoured.

A fundamental factor in the success of multimodal treatment is the degree of completion of the programme. Gustafsson et $a l^{14}$ have shown the existence of a doseresponse relationship and have highlighted the need to fulfil more than $70 \%$ of the items. In this sense, it is suggested that the more items of the programme are implemented, the better the patient's postoperative course. $^{15}$

In a recent study from our group, ${ }^{16}$ we were able to verify that despite having undergone training and having established an ERAS protocol in colorectal surgery, it was not fully implemented in daily clinical practice, with certain elements of the protocol having very low compliance, even in specialised centres. In this same study, which involved 2084 patients from 80 centres in our country, we found that an increase in compliance with evidencebased recommendations that constitute the PRI is associated with a decrease in postoperative complications.

As noted above, the evidence currently available concerns the benefits of such programmes in the immediate postoperative period, and there is still very little evidence as to whether the proper implementation of an ERAS protocol benefits patients in the long term. In this respect, Gustafsson et $a l^{17}$ analysed 5-year survival in a retrospective study and found that patients who were more compliant with an ERAS programme ( $\geq 70 \%$ of the protocol) exhibited a reduced risk of cancer-specific death at 5 years (HR: 0.58 ; $95 \%$ CI: 0.39 to 0.88 ). Other studies ${ }^{18-20}$ have addressed the relationship between ERAS programmes and overall and disease-free survival after colorectal surgery for cancer, although none have provided sufficient evidence to draw any firm conclusions. 
This study has been designed to support the working hypothesis that the correct implementation of an intensified recovery programme in patients undergoing colorectal surgery for cancer is related to better longterm oncological outcomes.

The primary objective is the analysis of survival at 5 years, overall survival, disease-related survival and disease-free survival.

The secondary objectives are to (1) evaluate the relationship between adherence to the protocol and 5-year survival, (2) analyse the importance of each item on survival and (3) evaluate the quality of life.

The data generated from this prospective, multicentre, observational cohort study will help to verify or better understand the suspected benefits of ERAS protocols regarding long-term survival in patients who have undergone colorectal surgery. The data will also help future research studies.

\section{Methods and analysis \\ Design}

A prospective, multicentre, observational cohort study in patients who meet the inclusion criteria.

\section{Setting}

This study will be conducted in 12 Spanish general hospitals, which were selected on the basis of having established an enhanced recovery protocol that complies with the recommendations of the Aragon Health Sciences Institute (IACS) and Spanish National Health Service (https:// portal.guiasalud.es/wp-content/uploads/2019/10/ viaclinica-rica_english.pdf). All hospitals selected have received prior standardised training according to the national plan for the implementation of intensified recovery in surgery (IMPRICA), also promoted by the IACS.

\section{Inclusion criteria}

All adult patients (aged $>18$ years) with a diagnosis of malignant colorectal cancer who are scheduled for radical surgery. Informed consent will be obtained from all subjects who will participate in the study voluntarily.

\section{Exclusion criteria}

Patient refusal, patients undergoing emergency surgery, patients under 18 years of age, patients diagnosed with stage IV cancer and existence of other concomitant surgical processes.

\section{Comparison groups}

As mentioned previously, the literature considers adequate implementation of a protocol to be compliance of more than $70 \%$. As such, two groups will be formed: one with more than $70 \%$ of the recorded items performed and the other with those that do not reach this percentage (table 1).

\section{Outcome measures}

The primary outcome measures are overall survival (patients alive from surgery to the last control), diseasefree survival (number of patients alive and without cancer recurrence from the intervention period until the end of follow-up) and disease recurrence (detected by CT or Flexible Colonoscopy (FCC), from the day of the intervention until the end of follow-up), which will also be studied.

Secondary outcome measures include compliance with individual protocol items and quality of life according to the EuroQol Five questionnaire.

\section{Follow-up}

The study is planned to start in September 2020, and for the survival study only patients with a minimum follow-up of 3 years will be considered. However, patients will still be recruited until the end of the 5-year period (September 2025) to allow study of the secondary objectives.

The follow-up plan is as follows: tumour markers (used to monitor colorectal neoplasia)-carcinoembryonic antigen determined at 3, 6, 9, 12, 15, 18, 21, 24, 30, 36, 42, 48 and 60 months and cancer antigen 19.9 determined at $3,6,9,12,15,18,21,24,30,36,42,48$ and 60 months; abdominal ultrasound performed at 3, 9, 15, 21, 36, 48 and 60 months; CT performed at 6, 12, 24, 36, 48 and 60 months; and complete colonoscopy performed at 2 and 5 years after intervention.

\section{Data collection and data management}

Data will be collected using an online data collection form via a secure, password-protected platform with predefined data fields at each centre. The variables to be collected are displayed in table 2. For the purpose of the study, we will record the following: complications at 60-day follow-up (surgical complications, infectious complications, cardiovascular complications), each rated as mild, moderate or severe and also according to the Clavien-Dindo classification; perioperative mortality (the number and percentage of deaths within 60 days of surgery); hospital stay, defined as the duration from the date of the end of surgery to the date of discharge from the hospital (in days); overall survival (the number and percentage of deaths that occur from the intervention to the end of follow-up); disease-free survival (the number of patients alive and with no cancer recurrence from the intervention period to the end of follow-up); and recurrence of the disease (detected by CT or FCC), from the day of the intervention until the end of follow-up.

The data collection platform Castor EDC (https://www. castoredc.com) will be used. This platform complies with all applicable laws and regulations. All identifiable data collected, processed and stored for the purposes of the project will be kept confidential at all times and comply with Good Clinical Practice guidelines for Research and the General Data Protection Regulation (Regulation (EU) 2016/679). 


\begin{tabular}{|c|c|c|}
\hline & Individual ERAS items included & Definitions of ERAS compliance for individual items included \\
\hline 1 & Presurgical education & Verbal and written ERAS education received at a dedicated preadmission visit \\
\hline 2 & Presurgical optimisation & $\begin{array}{l}\text { Patients stopped smoking } 4 \text { weeks before surgery, and alcoholics ceased all } \\
\text { alcohol consumption } 4 \text { weeks before surgery }\end{array}$ \\
\hline 3 & Preoperative fasting & $\begin{array}{l}\text { Preoperative fasting limited to } 2 \text { hours for clear liquids (water, coffee, juice without } \\
\text { pulp) and } 6 \text { hours for solids }\end{array}$ \\
\hline 4 & Patient blood management & $\begin{array}{l}\text { Set of measures applied to optimise preoperative haemoglobin, avoid bleeding } \\
\text { and avoid transfusion }\end{array}$ \\
\hline 5 & $\begin{array}{l}\text { Preoperative carbohydrate drink } \\
\text { preload }\end{array}$ & $\begin{array}{l}\text { Preoperative carbohydrate drink defined as at least } 50 \mathrm{~g} \text { of carbohydrate in at } \\
\text { least } 400 \mathrm{~mL} \text { of fluid, given in the form of a dedicated preoperative beverage with } \\
\text { a proven safety profile up until } 2 \text { hours before anaesthesia }\end{array}$ \\
\hline 6 & $\begin{array}{l}\text { Avoidance of long-acting sedative } \\
\text { premedication }\end{array}$ & $\begin{array}{l}\text { No long-acting sedative premedication given (eg, opioids, sedative antihistamines } \\
\text { and neuroleptics) }\end{array}$ \\
\hline 7 & Thromboprophylaxis & $\begin{array}{l}\text { Thromboprophylaxis (low-molecular-weight heparin and compression stockings) } \\
\text { given }\end{array}$ \\
\hline 8 & Antibiotic prophylaxis & Antibiotic prophylaxis given before skin incision \\
\hline 9 & Regional anaesthesia & $\begin{array}{l}\text { Anaesthetic procedure that allows rapid awakening, adequate analgesia } \\
\text { and patient recovery. This item is considered positive provided that any } \\
\text { major anaesthetic technique (spinal anaesthesia or general anaesthesia) is } \\
\text { accompanied by local or locoregional anaesthesia techniques, or continuous } \\
\text { epidural anaesthesia }\end{array}$ \\
\hline 10 & PONV prophylaxis & PONV prophylaxis given \\
\hline 11 & $\begin{array}{l}\text { Active prevention of unintentional } \\
\text { hypothermia }\end{array}$ & $\begin{array}{l}\text { Use of fluid heaters and/or thermal blanket for all patients during the surgical } \\
\text { procedure }\end{array}$ \\
\hline 12 & Goal-directed fluid therapy & $\begin{array}{l}\text { Intravenous fluid administration guided by haemodynamic goals based on the } \\
\text { cardiac output or derived monitoring by any validated cardiac output monitoring }\end{array}$ \\
\hline 13 & Laparoscopy or transverse incisions & $\begin{array}{l}\text { Laparoscopy is recommended, although this item will be considered positive in } \\
\text { those cases in which minimal incisions are used despite an open approach, }\end{array}$ \\
\hline 14 & Avoid drains & This item will be considered positive when no drains are left after closure \\
\hline 15 & Postoperative analgesia & $\begin{array}{l}\text { A multimodal analgesic management that includes at least two drugs to avoid or } \\
\text { reduce the administration of morphics }\end{array}$ \\
\hline 16 & Postoperative glycaemic control & $\begin{array}{l}\text { Patients receive glycaemic control in the first } 24 \text { hours, for target glycaemia } \\
<180 \mathrm{~g} / \mathrm{dL}\end{array}$ \\
\hline 17 & Early mobilisation & $\begin{array}{l}\text { Defined as the patient moved at least to an armchair in the first } 12 \text { postoperative } \\
\text { hours }\end{array}$ \\
\hline 18 & Early feeding & Defined as the patient tolerates oral feeding in the first 6 postoperative hours \\
\hline
\end{tabular}

ERAS, enhanced recovery after surgery; PONV, postoperative nausea and vomiting.

The research project will be monitored closely by a certified external auditor to ensure that study activities are carried out in accordance with the protocol, good clinical practice and applicable regulatory requirements. Local study documents can be selected for a local audit at participating hospitals. Data quality will also be audited.

\section{Statistical analysis}

According to the Postoperative Outcomes Within Enhanced Recovery After Surgery Protocol in Colorectal Surgery study that includes data from 82 Spanish hospitals, ${ }^{16}$ only $60 \%$ of centres with an ERAS protocol achieve more than $70 \%$ compliance. As such, we consider a scenario of seven high-compliance (HC) and five lowcompliance (LC) centres among the 12 hospitals that will collaborate with this research project. Estimating a difference in overall survival of around $10 \%$ (65\% LC vs $75 \%$ $\mathrm{HC}$ ), with a power of $80 \%$, a $95 \% \mathrm{CI}$ and $5 \%$ of potential losses, the required sample size is about 732 patients (366 in each group).

Given that the main objective (survival) may be subject to aspects inherent to each centre, irrespective of the intervention, it will be necessary to create comparable groups using the propensity score method (propensity score matching). A descriptive analysis of the data will be carried out. Qualitative variables will be represented by a frequency distribution of the percentages for each category, and quantitative variables will be explored using the Kolmogorov-Smirnov conformity test. The association 


\begin{tabular}{lll} 
Table 2 & Data variables collected & \\
\hline Patient & Surgical & Tumour \\
\hline Age & Surgical procedure & TNM (AJCC \\
Gender & Surgery time & classification) \\
BMI & Surgical approach & Grading (G1-G2-G3) \\
ASA score & Intraoperative blood & Grade of perineural \\
Smoking status & loss & or lymphatic \\
Hypertension & Resective surgery & invasion \\
Diabetes mellitus & (R0) & Margins \\
Coronary artery & Adjuvant treatment & Numbers of lymph \\
disease & & nodes studied \\
Stroke & & K-Ras \\
COPD/Asthma & & \\
Atrial fibrillation & & \\
$\begin{array}{l}\text { Peripheral arterial } \\
\text { disease }\end{array}$ & & \\
\hline
\end{tabular}

.AJCC, American Joint Committee on Cancer; ASA, American Society of Anesthesiologists physical status classification; BMI, body mass index; COPD, chronic obstructive pulmonary disease; TNM, tumour, node, metastases .

between factors will be investigated using hypothesis contrast tests, with a comparison of proportions when both variables are qualitative ( $\chi^{2}$, Fisher's exact test), a comparison of mean when one of them is quantitative (Student's t-test, analysis of variance (ANOVA), and the Mann-Whitney U test or the Kruskall-Wallis test if they do not follow a normal distribution) and a bivariate correlation (Pearson correlation coefficient) when both are quantitative or the Spearman correlation if the conditions for application of the former are not met. For comparisons in related samples when one of them is quantitative, Student's t-test and/or ANOVA will be used (Wilcoxon or Friedman's test if they do not follow a normal distribution). The analysis will be completed using multivariate regression models. A survival analysis will be performed using the Kaplan-Meier method, and the log-rank test will be used for survival comparisons between groups. Effects will be considered to be significant with a $p$ value of less than 0.05 .

\section{Patient involvement}

The study is supported by a patient advisory group which helped us with the patient's information material. This patient advisory group will meet on a regular basis for the duration of the study. At the end of the study, the patient advisory group will comment on the findings and contribute to the dissemination plan.

\section{Limitations of the study}

The limitations are those inherent to a prospective, non-randomised study, including difficulty in recruiting patients due to potential structural or multidisciplinary team problems and inappropriate number of patients in any of the arms due to a very high or very low level of compliance.

\section{ETHICS AND DISSEMINATION}

Ethical approval for this study was obtained from the Comité de Ética de la Investigación de la Comunidad Autónoma de Aragón (C.P.-C.I. PI20/086; on 4 March 2020). Local ethical approval is required at each participating centre. Although this study has no impact on clinical practice, informed consent will be requested from all participants. Patient data will be treated in accordance with the European General Data Protection Regulation 2016/679. The findings of this study are being submitted to peer-reviewed journals (BMJ Open, JAMA Surgery, Annals of Surgery, British Journal of Surgery). Abstracts will be submitted to relevant national and international meetings.

\section{Author affiliations}

${ }^{1}$ Lozano Blesa University Clinical Hospital, Zaragoza, Aragón, Spain

${ }^{2}$ Aragon Health Sciences Institute, Zaragoza, Aragón, Spain

${ }^{3}$ Miguel Servet University Hospital, Zaragoza, Aragón, Spain

${ }^{4}$ Miguel Hernandez University of Elche, Elche, Valenciana, Spain

${ }^{5}$ Health Service of Castilla-La Mancha, Guadalajara, Castilla-La Mancha, Spain

${ }^{6}$ Navarre Health Service, Pamplona, Navarra, Spain

${ }^{7}$ La Ribera Hospital, Alzira, Valenciana, Spain

${ }^{8}$ General University Hospital Gregorio Maranon, Madrid, Spain

${ }^{9}$ Hospital Universitario Infanta Leonor, Madrid, Spain

${ }^{10}$ Complejo Asistencial de Palencia, Palencia, Spain

${ }^{11}$ General University Hospital Consortium of Valencia, Valencia, Comunitat Valenciana, Spain

${ }^{12}$ Hospital Universitario Fundacion Jimenez Diaz, Madrid, Spain

${ }^{13}$ Hospital of Barbastro, Barbastro, Aragón, Spain

${ }^{14}$ Universidad Autonoma de Madrid, Madrid, Spain

Contributors J-MR-R proposed and designed the study, and is the main investigator. J-MR-R, JM-U, J-LM-R, J-RR-F, J-AG-E, JB-G, EDV-H, AA-G, MEC-R, CM-P, ML-A, E.E-G, ME-G, AMP-B, EM-T, AM-V, EB-R, DA-M, JMP-P, AA-M, ER-V, EB-P, HG-L, J R-M, CL-S, EC-DdL, LS-G, MC-O, JL-V, SO-L, JO-B, AA-S and DG-0 participated in the collaborators meeting, development of study concept and editing of protocol. All authors read and approved the final manuscript.

Funding The present research study was awarded a Ministerio de Ciencia e Innovación health research project grant (PI19/00291) from the Carlos III Institute of the Spanish National Health Service as part of the 2019 call for Strategic Action in Health.

Competing interests J-MR-R reports grants from Instituto de Investigacion Carlos III, during the conduct of the study. J-AG-E reports grants and personal fees from Vifor Pharma, Zambon and Sandoz, outside the submitted work. JR-M reports personal fees from Fresenius Kabi, Edwards Lifesciences, Dextera Medical and MSD, outside the submitted work

Patient consent for publication Not required.

Provenance and peer review Not commissioned; externally peer reviewed.

Open access This is an open access article distributed in accordance with the Creative Commons Attribution Non Commercial (CC BY-NC 4.0) license, which permits others to distribute, remix, adapt, build upon this work non-commercially, and license their derivative works on different terms, provided the original work is properly cited, appropriate credit is given, any changes made indicated, and the use is non-commercial. See: http://creativecommons.org/licenses/by-nc/4.0/.

ORCID iD

Jose-M Ramirez-Rodriguez http://orcid.org/0000-0001-7964-1166

\section{REFERENCES}

1 Siegel RL, Miller KD, Jemal A. Cancer statistics, 2019. CA A Cancer $J$ Clin 2019;69:7-34. 
2 American Cancer society. Survival rates for colorectal cancer, 2020. Available: https://www.cancer.org/cancer/colon-rectal-cancer/ detection-diagnosis-staging/survival-rates.html [Accessed 16 April 2020].

3 Artinyan A, Orcutt ST, Anaya DA, et al. Infectious postoperative complications decrease long-term survival in patients undergoing curative surgery for colorectal cancer: a study of 12,075 patients. Ann Surg 2015;261:497-505.

4 Straatman J, Cuesta MA, de Lange-de Klerk ESM, et al. Long-Term survival after complications following major abdominal surgery. $J$ Gastrointest Surg 2016;20:1034-41.

5 Snyder GL, Greenberg S. Effect of anaesthetic technique and other perioperative factors on cancer recurrence. $\mathrm{Br} J$ Anaesth 2010;105:106-15.

6 Yamaguchi K, Takagi Y, Aoki S, et al. Significant detection of circulating cancer cells in the blood by reverse transcriptasepolymerase chain reaction during colorectal cancer resection. Ann Surg 2000;232:58-65

7 Wang J-Y, Wu C-H, Lu C-Y, et al. Molecular detection of circulating tumor cells in the peripheral blood of patients with colorectal cancer using RT-PCR: significance of the prediction of postoperative metastasis. World J Surg 2006;30:1007-13.

8 Shakhar G, Ben-Eliyahu S. Potential prophylactic measures against postoperative immunosuppression: could they reduce recurrence rates in oncological patients? Ann Surg Oncol 2003;10:972-92.

9 Mafune K, Tanaka Y. Influence of multimodality therapy on the cellular immunity of patients with esophageal cancer. Ann Surg Oncol 2000;7:609-16.

10 Gonzalez-Ayora S, Pastor C, Guadalajara H, et al. Enhanced recovery care after colorectal surgery in elderly patients. compliance and outcomes of a multicenter study from the Spanish Working group on eras. Int J Colorectal Dis 2016;31:1625-31.

11 Martínez AB, Longás J, Ramírez JM, Ramirez-Rodriguez JM. A model for lymphocyte activation in open versus laparoscopic surgery in colorectal cancer patients in enhanced recovery after surgery (ERAS) protocols. Int J Colorectal Dis 2017;32:913-6.

12 Scott MJ, Baldini G, Fearon KCH, et al. Enhanced recovery after surgery (ERAS) for gastrointestinal surgery, part 1: pathophysiological considerations. Acta Anaesthesiol Scand 2015;59:1212-31.

13 Schricker T, Lattermann R. Perioperative catabolism. Can J Anesth/J Can Anesth 2015;62:182-93.

14 Gustafsson UO, Scott MJ, Schwenk W, et al. Guidelines for perioperative care in elective colonic surgery: enhanced recovery after surgery (ERAS $₫$ ) Society recommendations. Clin Nutr 2012;31:783-800.

15 Pedziwiatr M, Kisialeuski M, Wierdak M, et al. Early implementation of Enhanced Recovery After Surgery (ERAS $®$ ) protocol - Compliance improves outcomes: A prospective cohort study. Int J Surg 2015;21:75-81.

16 Ripollés-Melchor J, Ramírez-Rodríguez JM, Casans-Francés R, et al. Postoperative outcomes within enhanced recovery after surgery protocol in colorectal surgery (power study). JAMA Surgery 2019;154:725-36.

17 Gustafsson UO, Oppelstrup H, Thorell A, et al. Adherence to the eras protocol is associated with 5-year survival after colorectal cancer surgery: a retrospective cohort study. World J Surg 2016;40:1741-7.

18 Slieker J, Frauche P, Jurt J, et al. Enhanced recovery eras for elderly: a safe and beneficial pathway in colorectal surgery. Int $J$ Colorectal Dis 2017;32:215-21.

19 Slim K, Regimbeau JM, Francophone Group for Enhanced Recovery After Surgery (GRACE). Increased survival might be an unexpected additional advantage of enhanced recovery after surgery programs. $J$ Visc Surg 2018;155:169-71.

20 Asklid D, Segelman J, Gedda C, et al. The impact of perioperative fluid therapy on short-term outcomes and 5-year survival among patients undergoing colorectal cancer surgery - A prospective cohort study within an ERAS protocol. Eur J Surg Oncol 2017;43:1433-9. 